\title{
Prevalence of Intestinal Parasitic Infections Among Patients with Diarrhea at Wonago Health Center, Southern Ethiopia: A Retrospective Study
}

\author{
Girum Tefera \\ Department of Medical Biotechnology, Tehran University of Medical Sciences, Iran
}

Copyright (C) 2015 Horizon Research Publishing All rights reserved.

\begin{abstract}
Background: Intestinal parasitic infections are among the most common infections worldwide. High prevalence is found in people with low socio-economic status, poor living condition, overcrowded areas, poor environmental sanitation, improper garbage disposal, unsafe water supply and unhygienic personal habits. Thus, the objective of this retrospective study was to determine the prevalence of intestinal parasitosis among patients with diarrhea who visited Wonago health center Southern Ethiopia. Methodology: Institution based retrospective study design was employed. Stool examination records of all patients with diarrhea who visited Wonago health center from September 2010 to September 2012 were included. A total of 10694 stool examination results of those diarrheic patients were analyzed for this study. Samples were examined using direct saline wet mount technique. Statistical analysis was done by SPSS version 16 and results were shown in percent and prevalence rates. Results: The overall prevalence of intestinal parasite for at least one parasite in this retrospective study was 1776/10694(16.6\%). Furthermore, $5.7 \%$ of those positive patients were infected by two species of parasites and $4.6 \%$ of patients had triple infections. The most prevalent parasites were $E$. histolytica/dispar 956/1776(53.8\%) followed by G. lamblia 420/1776(23.7\%) and Taenia species 140/1776(7.9\%) respectively. Conclusion: Intestinal parasitosis is highly prevalent in varying magnitude among diarrheic patients who visited Wonago health Center, Southern Ethiopia. E. histolytica/dispar was the most prevalent parasite infecting patients followed by, G. lamblia, Taenia species and H. nana. Because of absence of molecular diagnosis for E. histolytica, over diagnosis might be possible for the high prevalence of amoebiasis.
\end{abstract}

Keywords Intestinal Parasitosis, Prevalence, Protozoa, Helminthes, Wonago Health Center

\section{Introduction}

Human intestinal parasitic infections are among the most common infections in the world and are responsible for considerable morbidity and mortality (1). Apart from causing mortality and morbidity, infection with intestinal parasites has been associated with malnutrition, stunting of linear growth, mental function, verbal ability, physical weakness and low educational achievement in school children (2). Furthermore, chronic intestinal parasitic infections have become the subject of speculation and investigation in relation to the spreading and severity of other infectious diseases of viral origin, tuberculosis and malaria (3). The level of harm caused by intestinal parasite infection to the health of individual and communities depend on: the parasite species, the nature of the interaction between the parasite and the concurrent infections, the intensity and course of infection and nutritional and immunological status of the population $(3,4)$. It is estimated that 3.5 billion people are affected globally and 450 million are ill as a result of these infections. Among the infections responsible for considerable morbidity and mortality in the world, more than 1.2 billion people are infected with Ascaris lumbricoides; 740 million people with hookworms; 795 million with Trichuris trichiura and 300 million with Enterobius vermicularis (5). The epidemiology of intestinal parasitic infections shows that these parasites are found in every age group and in both sexes. However, the incidence is high in some areas and in some age groups (6). Studies carried out in different countries have shown that the situation of an individual is an important cause in the prevalence of intestinal parasitic infection, having a greater rate in children (7). High prevalence is found in people with low socio-economic status, poor living condition, overcrowded areas, poor environmental sanitation, improper garage disposal, unsafe water supply and unhygienic personal habits. These factors are the cause of major proportion of burden of the disease and death in developing countries $(8,9)$. In sub-Saharan African countries; up to 250 million people are estimated to be infected with at least one or more species of intestinal nematodes (10). In Ethiopia, few studies were conducted among different community to indicate the prevalence of intestinal parasitosis. Study Conducted on 
urban dwellers in south west Ethiopia has indicated an overall prevalence of $83 \%$. The most prevalent intestinal parasites identified were $\mathrm{T}$. trichiura $(16.4 \%)$, A. lumbricoides (5.8\%) and S. mansoni (1.5\%) (11). Intestinal parasitosis could be highly prevalent in Ethiopia because of poor socioeconomic standards, poor environmental sanitation and ignorance of simple health promoting factors like hand washing practices, use of latrine and consumption of raw/undercooked vegetables or dairy and beef products (12). Although there are few studies conducted to assess the situation of intestinal parasitosis in different parts of Ethiopia, there are still several localities for which epidemiological information is not available including the present study area. Therefore, this retrospective study was aimed to assess the prevalence of intestinal parasitosis among diarrheic patients visited Wonago health center, southern, Ethiopia.

\section{Methods and Materials}

\subsection{The Study Area}

Wonago Woreda ( $6^{\circ} 20^{\prime}$ and E $38^{\circ} 19^{\prime}$ ) is located 380 $\mathrm{km}$ from Addis Ababa in Gedeo Zone, Southern Nations, Nationalities and Peoples Regional State (SNNPR) and bordering with Oromia to the west and northwest, Yirgachefee to the south and southeast, Dilla to the north and Bule to the east. It is approximately $248 \mathrm{sq} . \mathrm{km}(24,790 \mathrm{ha})$ and comprises of 19 Kebeles (Fig. 1).

The 2005 census indicates that Wonago Woreda has a total population of 162,663 of which $78,649(48.3 \%)$ are males and $84,014(51.6 \%)$ are females. The population density of the Woreda is 702 persons per $\mathrm{km} 2$ at a national growth rate of 1.07 percent. Seventy four percent of the population in the Woreda are the Gedeo people.

The present retrospective study was conducted at Wonago health Center in Wonago town, Southern Ethiopia. Institution based retrospective study design was used and the data's of stool examinations of all patients those with diarrhea who visited the health center from September 2010 to September 2012 were considered for the study. All patients those with diarrhea were sent to the laboratory for stool parasitological examination. Within this time frame, stool examinations were done for a total of 10694 patients and all the results were analyzed for the purpose of this study. Proper stool samples were collected throughout the year with labeled clean, dry and sterile plastic container. Direct stool examinations were done using saline wet mount technique within less than 20 minutes of stool sample collection. All stool samples were examined only with direct saline method primarily because of the lengthy procedures of concentration technique which is not routinely done in health care institutions. After stool examinations were completed, infected patients were treated based on the identified parasite species. Data entry and analysis was done using SPSS version 16 computer software. Data was summarized and presented in a descriptive measure such as a table, mean and percent. $\mathrm{P}$ value less than 0.05 was considered statistically significant. Records of patients were manipulated only by researcher. Appropriate treatment was given to those patients who were positive for intestinal parasitic infection by physicians. All patients involved in this report were provided a written informed consent prior to data collection. Furthermore, written informed consent was obtained from each patient for publication.

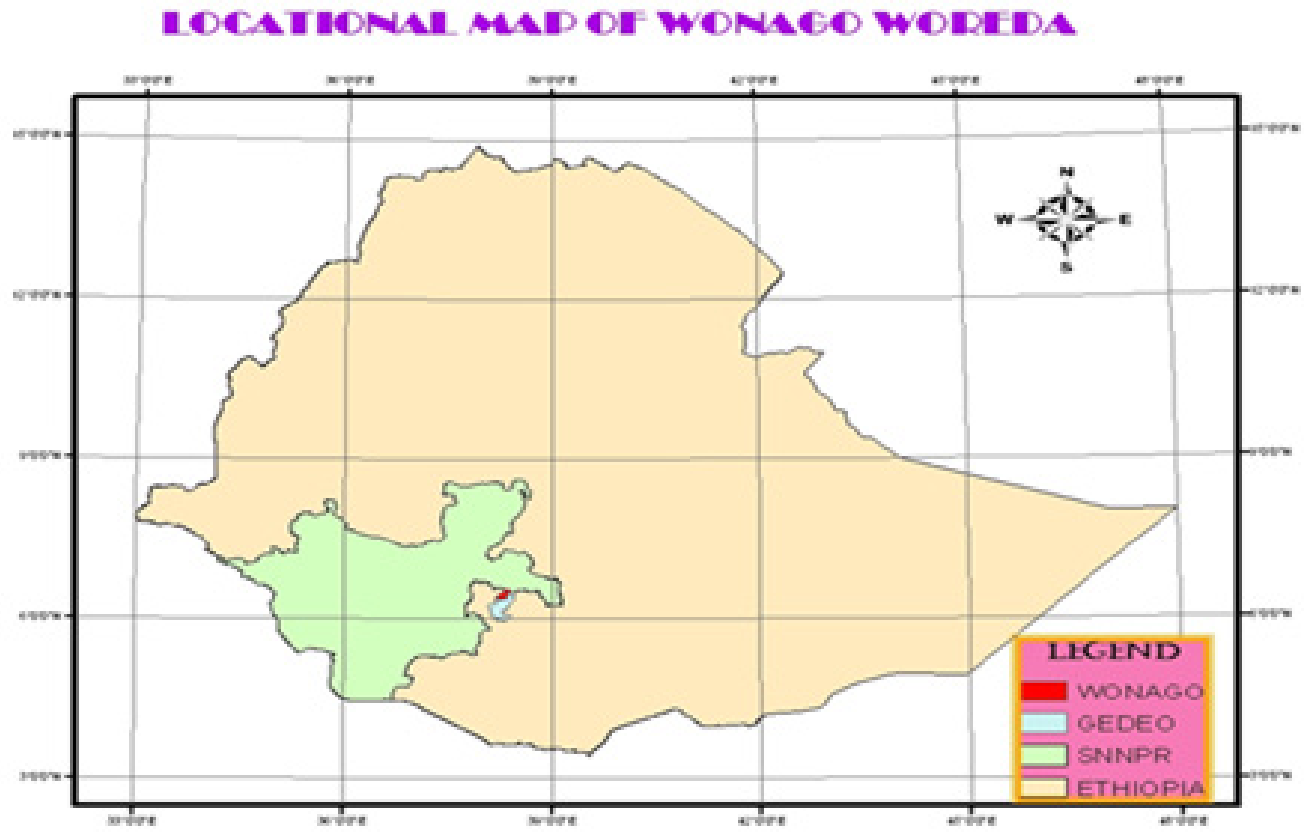




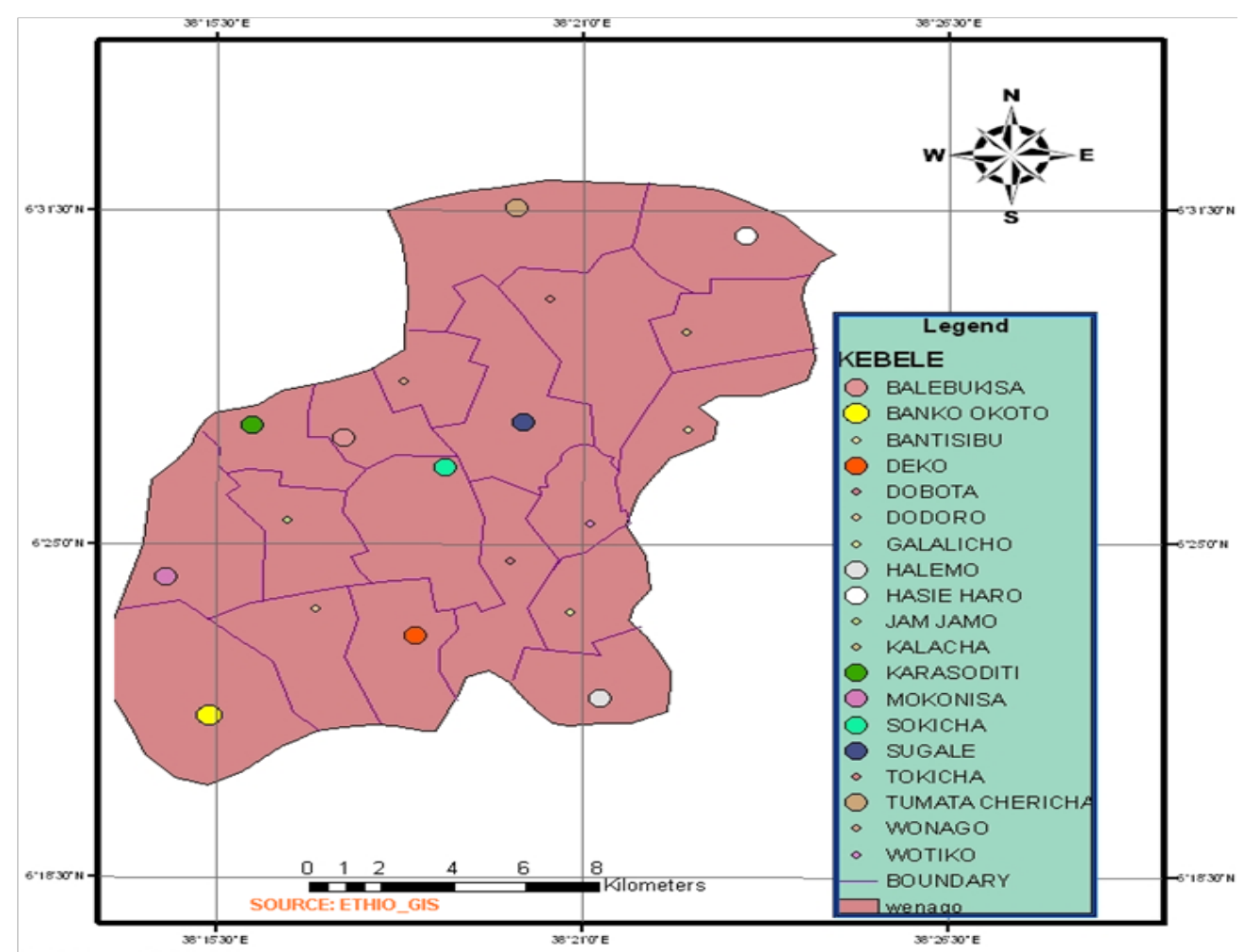

Figure 1. Location of Wonago Woreda in Gedeo zone; Southern Nations, Nationalities and Peoples Regional State (SNNPR).

\section{Result}

A total of 10694 diarrheic patients stool examination results were included for the purpose of this retrospective study. The age of patients' ranges from six month to ninety two years and $30 \%$ of the study population were children under the age of 15 years. Majority $(76 \%)$ of patients was under the age of 30 years and female to male ratio of the patients were 1.3:1.Microscopic stool sample examinations had showed that infections with various intestinal helminthes and protozoan parasites were common in all age groups of patients. In this study, thirteen (13) species of parasites were identified. The overall intestinal parasite prevalence for at least one parasite in this study was $1776 / 10694$ (16.6\%). Furthermore, 102/1776(5.7\%) of those positive patients were infected by two species of parasites and 4/1776(2.3\%) of patients had triple infections. The most prevalent parasites were E. histolytica/dispar trophozoite and cyst stage 956/1776(53.8\%) followed by G. lamblia 420/1776(23.7\%) trophozoite and cyst stage and Taenia species 140/1776(7.9\%) respectively. Moderately prevalent parasites were $H$. nana 94/1776(5.3\%), E. vermicularis $38 / 1776(2.1 \%)$, A. lumbricoides 36/1776(2.0\%) and S. stercolaris $24 / 1776(1.4 \%)$. The least prevalent parasites were Hookworms 16/1776(0.9\%), both T. trichiura and E. coli infected $14 / 1776(0.8 \%)$ patients each, T. hominis 12/1776(0.7\%), both S. mansoni and Trichostrongyloide species infected 6/1776(0.3\%) patients each. $986 / 1776(55.5 \%)$ of those patients with intestinal parasitosis were female patients and Taenia species, E. hitolytica/dispar and G. lamblia were higher in females than males (Table1)

Table 1. Distribution of each species of parasite based on gender among patients with diarrhea at Wonago health center, Northern, Ethiopia (Sept. 2010-Sept. 2012)

\begin{tabular}{|c|c|c|c|}
\hline \multirow{2}{*}{ Parasite species } & \multicolumn{3}{|c|}{$\begin{array}{c}\text { Parasite distribution among } \\
\text { paitents,Total=10694 }\end{array}$} \\
\cline { 2 - 4 } & $\begin{array}{c}\text { Male, } \\
(\mathrm{N}=2592, \%)\end{array}$ & $\begin{array}{c}\text { Female } \\
(\mathrm{N}=6102, \%)\end{array}$ & Total (\%) \\
\hline E. histolytica/dispar & $434(48.8)$ & $522(59.8)$ & $956(107.6)$ \\
\hline G. lamblia & $188(21.6)$ & $232(26.2)$ & $420(47.4)$ \\
\hline E. coli & $8(1)$ & $6(0.6)$ & $14(1.6)$ \\
\hline T. hominis & $2(0.2)$ & $10(1.2)$ & $12(1.4)$ \\
\hline A. lumbricoides & $16(1.8)$ & $20(2.2)$ & $36(4)$ \\
\hline Hookworms & $10(1.2)$ & $6(0.6)$ & $16(1.8)$ \\
\hline S. stercolaris & $14(1.6)$ & $10(1.2)$ & $24(2.8)$ \\
\hline T. trichiura & $2(0.2)$ & $12(1.4)$ & $14(1.6)$ \\
\hline H. nana & $48(5.4)$ & $46(5.2)$ & $94(10.6)$ \\
\hline E. vermicularis & $22(2.4)$ & $16(1.8)$ & $38(4.2)$ \\
\hline Taenia species & $40(4.6)$ & $100(11.2)$ & $140(15.8)$ \\
\hline S. mansoni & $4(0.4)$ & $2(0.2)$ & $6(0.6)$ \\
\hline $\begin{array}{c}\text { Trichostrongyloide } \\
\text { species }\end{array}$ & $2(0.2)$ & $4(0.4)$ & $6(0.6)$ \\
\hline Total & $790(89)$ & $986(111)$ & 1776 \\
\hline
\end{tabular}

Table 2 shows that the distribution of each species of parasite among patients in age group interval and gender. $1376 / / 1776(77.5 \%)$ of patients were infected with E. hitolytica/dispar and G. lamblia and they were highly 
prevalent across all age groups. They were the most prevalent protozoal parasites in patients under the age of 15 years $(223 ; 25.1 \%)$. H. nana, A. lumbricoides and Hookworms were also among the commonest helminthic parasite species in this age group ( $<15$ years). However, Taeniasis was highly prevalent among adults typically in the age groups 16-30 and 31-45 years. In most age groups, the numbers of parasite infected female patients were higher than males (Table 2). The most prevalent double intestinal infection observed was E. histolytica/dispar and G. lamblia (29 patients; 3.3\%), H. nana and E. histolytica/dispar (3 patients; $0.3 \%$ ), G. lamblia and $\mathrm{H}$. nana (3 patients; $0.3 \%$ ) and Taenia species and E. histolytica/dispar (2 patients; $0.3 \%)$.

Table 2. Distribution of intestinal parasites among patients with diarrhea in different age groups and gender at Wonago health Center, Northern, Ethiopia (Sept. 2010-Sept. 2012), (Note: $\mathrm{M}=$ Male, $\mathrm{F}=$ Female, yr. = Year)

\begin{tabular}{|c|c|c|c|c|c|c|c|c|c|c|}
\hline & & \multicolumn{8}{|c|}{$\begin{array}{l}\text { Parasite infected patients in number by age group } \\
\qquad(\mathrm{N}=10694)\end{array}$} & \multirow{3}{*}{ Total } \\
\hline \multirow{2}{*}{\multicolumn{2}{|c|}{ Parasite species }} & \multicolumn{2}{|c|}{$<15 \mathrm{yr}$} & \multicolumn{2}{|c|}{$16-30 \mathrm{yr}$} & \multicolumn{2}{|c|}{$31-45 \mathrm{yr}$} & \multicolumn{2}{|c|}{$>46 \mathrm{yr}$} & \\
\hline & & M & $\mathrm{F}$ & $\mathrm{M}$ & $\mathrm{F}$ & M & $\mathrm{F}$ & $\mathrm{M}$ & $\mathrm{F}$ & \\
\hline \multirow{2}{*}{$\begin{array}{c}\text { E.histoytica } \\
\text { /dispar }\end{array}$} & Trophozoite & 148 & 120 & 134 & 182 & 46 & 80 & 44 & 66 & 820 \\
\hline & Cyst & 16 & 6 & 22 & 40 & 6 & 18 & 18 & 10 & 136 \\
\hline \multirow{2}{*}{ G.lamblia } & Trophozoite & 90 & 60 & 44 & 74 & 20 & 28 & 26 & 36 & 378 \\
\hline & Cyst & - & 6 & 2 & 18 & 2 & 4 & 4 & 6 & 42 \\
\hline \multicolumn{2}{|c|}{ E.coli } & 4 & - & 2 & - & - & 2 & 2 & 4 & 14 \\
\hline \multicolumn{2}{|c|}{ T.hominis } & - & 2 & - & - & - & 2 & 2 & 6 & 12 \\
\hline \multicolumn{2}{|c|}{ A. lumbricoides } & 10 & 4 & 4 & 12 & - & 4 & 2 & - & 36 \\
\hline \multicolumn{2}{|c|}{ Hookworms } & 8 & 2 & 2 & 2 & - & - & - & 2 & 16 \\
\hline \multicolumn{2}{|c|}{ S. stercolaris } & 2 & - & 4 & 2 & 4 & 2 & 4 & 6 & 24 \\
\hline \multicolumn{2}{|c|}{ T. trichiura } & - & - & 2 & 8 & - & 2 & - & 2 & 14 \\
\hline \multicolumn{2}{|c|}{ H. nana } & 38 & 32 & 4 & 10 & 4 & 4 & 2 & - & 94 \\
\hline \multicolumn{2}{|c|}{ E. vermicularis } & 6 & 4 & 10 & 6 & 6 & 6 & - & - & 38 \\
\hline \multicolumn{2}{|c|}{ Taenia species } & 14 & 2 & 12 & 60 & 6 & 22 & 8 & 16 & 140 \\
\hline \multicolumn{2}{|c|}{ S. mansoni } & 2 & - & 2 & 2 & - & - & - & - & 6 \\
\hline \multicolumn{2}{|c|}{ Trichostrongyloide species } & 2 & - & - & 2 & - & 2 & - & - & 6 \\
\hline \multicolumn{2}{|c|}{ Total } & 340 & 238 & 244 & 418 & 94 & 176 & 112 & 154 & 1776 \\
\hline
\end{tabular}

Table 3. Double parasitic infections (poly parasitism) among patients with diarrhea who visited Wonago health Center, Northern, Ethiopia (Sept. 2010-Sept. 2012) One patient was infected by three species of parasites (A. lumbricoides, T. trichiura and S. mansoni ) and another one patient has a triple infection by Hookworms, G. lamblia and H. nana (Table3)

\begin{tabular}{|c|c|c|}
\hline Parasite Species in Double & Frequency $(\mathrm{N}=1776)$ & $\%$ \\
\hline E. histolytica/dispar and G. lamblia & 58 & 6.6 \\
\hline G. lamblia and H. nana & 8 & 1 \\
\hline A. lumbricoides and S. mansoni & 8 & 1 \\
\hline A. lumbricoides and Taenia species & 4 & 0.4 \\
\hline $\begin{array}{c}\text { Taenia species and } E . \\
\text { histolytica/dispar }\end{array}$ & 4 & 0.4 \\
\hline H. nana and $E$. vermicularis & 4 & 0.4 \\
\hline $\begin{array}{l}\text { A. lumbricoides and } E \text {. } \\
\text { histolytica/dispar }\end{array}$ & 2 & 0.2 \\
\hline Hookworms and E. histolytica/dispar & 2 & 0.2 \\
\hline $\begin{array}{c}\text { E. histolytica/dispar and } E \text {. } \\
\text { vermicularis }\end{array}$ & 2 & 0.2 \\
\hline Hookworms and G. lamblia & 2 & 0.2 \\
\hline G. lamblia and S. stercolaris & 2 & 0.2 \\
\hline S. stercolaris and T. hominis & 2 & 0.2 \\
\hline E. coli and T. hominis & 2 & 0.2 \\
\hline
\end{tabular}




\section{Discussion}

Intestinal parasitic infections are amongst the most common widespread of all chronic human infections worldwide. The present retrospective study aimed at determining the prevalence of different intestinal parasitic infections among diarrheic patients who visited Wonago health Center, for a period of two years (Sept. 2010- Sept. 2012). In this study the overall prevalence of intestinal parasites was 1776/10694 (16.6\%) which was much lower than the previous studies conducted in different parts of Ethiopia; Jimma (83\%) by Amare et al., Gondar (52.6\%) by Getachew et al. and Hawassa (55.0\%) by Shimelis et al. (11, $13,14)$ and other countries; Vietnam $(88.0 \%)$ by Verl et al. and Nigeria $(52 \%)$ by Morenikeji et al. $(15,16)$. The result of this study is almost similar with the study conducted in Kashmir (18.02\%) by Tariq Masood Malik (17). In contrast, the present study is very much higher when compared with the study conducted in Greek (12.02\%) by Papazahariadou et al. (18). These differences in prevalence could be due to the place and living standard of study subjects or due to a reflection of the local endemicity and geographic condition of the study area. The low sensitivity of direct saline method could also be responsible for low prevalence in this study. E. histolytica/dispar (53.8\%) was the highly prevalent of protozoan parasite in the present study which was higher as compared to the study done in different localities of Ethiopia; Jimma (5.6\%), Gondar town (10.3\%) and north Gondar (27.3\%) and other countries; Myanmar district (6.2\%), Parma Italy (4.1\%), Thai (7.1\%) and Kuala Lumpur (0.4\%) $(11,13,19,20,21,22,23)$. However, the prevalence in the present study was lower than in Kenya which was (74.1\%) (24). Higher prevalence of E. histolytica/dispar in this study might be due to low sanitary condition, contamination of drinking water or vegetables. The high prevalence might also be due to E. histolytica over diagnosis because of absence of molecular based diagnosis to differentiate E. histolytica from E. dispar . G. lamblia (23.7\%) was the second most prevalent intestinal parasites in this study which was higher than the study conducted in different part of Ethiopia; Jimma (18.2\%), Gondar (3.8\%) and other countries; Myanmar district (4.5\%), Parma Italy (1.6\%) and Cameroon $(0.6 \%)(6,11,13,19,20)$. In contrast, it is lower than one study conducted in Delgi village children, northern Gondar Ethiopia which was $41.9 \%$ (22). The higher prevalence in this study is might be because of drinking water contamination and low socioeconomic status of study population. Taenia species $(7.9 \%)$ and $\mathrm{H}$. nana $(5.3 \%)$ were the third and fourth most prevalent parasites in this retrospective study respectively. Especially, the result of Taenia species in this study was higher than other studies conducted in Jimma Ethiopia (5.8\%) (22) However, it was lower than the findings of Mengistu Legesse and Berhanu Erko around Lake Langano, Ethiopia (13.9\%) (25). Habit of eating raw and roasted (under cooked) meat in Ethiopia plays an important role for the higher prevalence rate of Taeniasis. H. nana infection was higher in children in this study and was higher when compared with the study of
Alemeshet et al in rural areas of Jimma Zone Ethiopia (2.1\%) (26). However, it was lower than the study of Amare et al. in Jimma town Ethiopia (20.5\%) and among Thai people (7.8\%) $(11,21)$. In all cases, the high prevalence of $\mathrm{H}$. nana is linked to its direct transmission and low hygienic status of children. The magnitude of the remaining intestinal parasitic infections in this retrospective study varies and/or comparable with other study areas. The differences in prevalence rate between the study areas might be due to culture, practice, living standard and category of the study population in addition to the period of study and the methods employed for stool examination. In areas where numerous kinds of intestinal parasites found, polyparasitism infections were common. In this study single, double and triple infection (16.6\%, 5.7\% and $2.3 \%$ ) were respectively observed. E. histolytica/dispar and G. lamblia (3.3\%) was the most frequent double infection. Triple infections were occurred by A. lumbricoides, T. trichiura and S. mansoni and Hookworms, G. lamblia and H. nana in the present study. This may be probably due to environmental conditions and methods of transmission that was favorable for parasites live together for the occurrence of high prevalence of the two parasites in this study.

\section{Conclusions}

Intestinal parasitosis was highly prevalent in varying magnitude among diarrheic patients who visited Wonago health Center for the past two year (Sept. 2010-Sept.2012). E. histolytica/dispar was the most prevalent parasite infecting patients followed by G. lamblia, Taenia species and H. nana. The absence of molecular diagnosis for E. histolytica, over diagnosis might be possible for high prevalence amoebiasis in thisnstudy.

\section{REFERENCES}

[1] Kongs A, Marks G, Verle P, Van Der, Stuyft P (2001) The Unreliability of the Kato-Katz Technique limits its Usefulness for Evaluating S. mansoni Infections. Trop Med Inter Heal 6: 163-169.

[2] Nokes C, Bundy DAP (1994) Does helminth infection affect mental processing and educational achievement? Parasitol Today 10: 14-18.

[3] Wolde-michael T, Assefa T, Seyom S (1990) Intestinal parasitism among the student population of the wanji showa sugar state. Ethiop J Heal Dev 4: 45-49.

[4] Kloos H, Tesfa-Yohannes T (1993) "Intestinal parasitism in Ethiopia. The ecology of health and disease in Ethiopia". Oxford: West veiw press,223-235.

[5] World Health Organization (1991) Basic laboratory methods in medical parasitology. WHO, Geneva, Switzerland 32.

[6] Judith V Mbuh, Helen N Ntonifor, James T Ojong (2010) The 
incidence, intensity and host morbidity of human parasitic protozoan infections in gastrointestinal disorder out patients in Buea Sub Division, Cameroon. J Infect Dev Ctries 4: $38-43$.

[7] Udonsi JK, Amabibi MI (1992) The human environment occupation and possible water born transmission of Hookworm in endemic coastal communities of Niger delta, Nigeria. Pubilic Health 106: 63-71.

[8] Chan MS (1997) The global burden of intestinal nematode infectionsfifty years on. Parasitol Today 13: 438-443.

[9] De Silva NR, Guyatt HL, Bundy DA (1997) Morbidity and mortality due to ascaris-induced intestinal obstruction. Trans R Soc Trop Med Hyg 91: 31-36.

[10] World Health Organization (2002) Prevention and control of schistosomiasis and soil-transmitted helminthiasis. WHO, Technical Report Series 912.

[11] Amare Mengistu, Solomon Gebre-Selassie, Tesfaye Kassa (2007) Prevalence of intestinal parasitic infections among urban dwellers in southwest Ethiopian. Ethiop J Heal Dev 21: 12-17.

[12] Yeneneh H (1994) Survey of intestinal parasites in Bureau area Southwestern Ethiopia. Ethiop J Heal Dev 8: 29-35.

[13] Getachew Hailemariam, Afework Kassu, Gemeda Abebe, Eba Abate, Demekech Damte et al. (2004) Intestinal parasitic infections in HIV/ AIDS and HIV seronegative individuals in a teaching hospital, Ethiopia. Jpn J Infect Dis 57: 41-43.

[14] Shimelis Assefa, Berhanu Erko, Girmay Medhin, Zelalem Assefa, Techalew Shimelis (2009) Intestinal parasitic infections in relation to HIV/AIDS status, diarrhea and CD4 T-cell count. BMC Infectious Diseases 9.

[15] Verle P, Kongs A, De NV, Thieu NQ, Depraetere K et al. (2003) Prevalence of intestinal parasitic infections in northern Vietnam. Trop Med Intern Heal 8: 961-964.

[16] Morenikeji OA, Azubike NC, Ige AO (2009) Prevalence of intestinal and vector-borne urinary parasites in communities in south-west Nigeria. J Vector Borne Dis 46: 164-167.

[17] Tariq Masood Malik, Zahid Farooq Baig (2006) Frequency and Pattern of Intestinal Parasitic Infestations in upper Neelum Valley. Pak Armed Forces Med J 4: 67-73.

[18] Papazahariadou MG, Papadopoulos EG, Frydas SE, Mavrovouniotis Ch, Constantinidis TC, et al. (2004) Prevalence of gastrointestinal parasites in the Greek population: local people and refugees. Ann Gastroentrol 17: 194-198.

[19] Prasert R, Pitak W, Nipon T (2008) Factors Associated with IntestinalParasites among Households in Ratchaburi Province, Thai-Myanmar Border Area. J Trop Med Parasitol 31: 85-94.

[20] Simona Peruzzi, Chiara Gorrini, Giovanna Piccolo, Adriana Calderaro, Giuseppe Dettori, et al. (2006) Prevalence of intestinal parasites in the area of Parma during the year 2005. Acta biomed 77: 147-151.

[21] Chukiat Sirivichayakul, Chanathep Pojjaroen-anant, Pataraporn Wisetsing, Chutatip Siripanth, Pornthep Chanthavanich, et al. (2003) Prevalence of Intestinal Parasitic Infection among Thai People with Mental Handicaps. Southeast Asian J Trop Med Pub Heal 34: 259-263.

[22] Asrat Ayalew, Tewodros Debebe, Alemayehu Worku (2011) Prevalence and risk factors of intestinal parasites among Delgi school children, North Gondar, Ethiopia. J Parasitol Vect Biol 3: 75-78.

[23] Jamaiah I, Rohela M (2005) Prevalence of Intestinal Parasites among Members of the Ppublic in Kuala Lumpur, Malaysia. Southeast Asian J Trop Med Pub Heal 36: 68-71.

[24] Robert M Nyarango, Peninah A Aloo, Ephantus W Kabiru, Benson O Nyanchongi (2008) The risk of pathogenic intestinal parasite infections in Kisii Municipality, Kenya. BMC Publ Heal 8.

[25] Mengistu Legesse, Berhanu Erko (2004) Prevalence of intestinal parasites among school children in a rural area close to the southeast of Lake Langano, Ethiopia. Ethiop J Heal Dev 18: 116-121.

[26] Alemeshet Yami, Yoseph Mamo, Seleshi Kebede (2011) Prevalence and Predictors of Intestinal Helminthiasis among School Childern in Jimma Zone; A Cross-Sectional Study. Ethiop J Heal Sci 21: 167-174. 